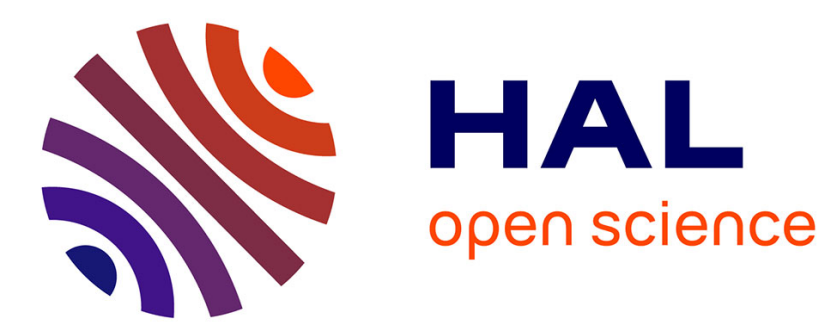

\title{
ÉTHIQUE ET POLITIQUE DES LARMES DANS VIRGINIE DE CAMPISTRON
}

\author{
Sophie Marchand
}

\section{To cite this version:}

Sophie Marchand. ÉTHIQUE ET POLITIQUE DES LARMES DANS VIRGINIE DE CAMPISTRON. Littératures classiques (continuation des Cahiers de littérature du XVIIe siècle), 2004, Campistron et consorts : tragédie et opéra en France (1680-1733), 52, pp.193-206. hal-03310890

\section{HAL Id: hal-03310890 https://hal.sorbonne-universite.fr/hal-03310890}

Submitted on 30 Jul 2021

HAL is a multi-disciplinary open access archive for the deposit and dissemination of scientific research documents, whether they are published or not. The documents may come from teaching and research institutions in France or abroad, or from public or private research centers.
L'archive ouverte pluridisciplinaire HAL, est destinée au dépôt et à la diffusion de documents scientifiques de niveau recherche, publiés ou non, émanant des établissements d'enseignement et de recherche français ou étrangers, des laboratoires publics ou privés. 


\title{
ÉTHIQUE ET POLITIQUE DES LARMES DANS VIRGINIE DE CAMPISTRON
}

\author{
Sophie Marchand
}

Considérée par la critique comme une œuvre de jeunesse sans grande originalité, Virginie, première tragédie de Campistron, demeure trop souvent négligée. Les Frères Parfaict implorent l'indulgence du lecteur en faveur de la jeunesse de l'auteur'. Quant à La Porte, il rêve à ce que serait cette tragédie si Campistron l'avait composée dix ans plus $\operatorname{tard}^{2}$. L'œuvre de Campistron souffre par ailleurs des rapprochements que ses commentateurs ne cessent d'effectuer avec celles de ses aînés, en particulier Racine et Corneille. D'Alembert ne manque pas de souligner que Virginie comme Arminius, toutes deux "pièces faiblement écrites et un peu trainnantes ", bénéficièrent des conseils de Racine ${ }^{3}$. Plus près de nous, Lancaster estime pour sa part que les pièces de jeunesse de Campistron doivent davantage à Corneille qu'à Racine. Ainsi, Virginie, mettant en scène un sujet tiré de l'histoire romaine, illustrant l'hérö̈sme du personnage éponyme et reprenant un certain nombre de vers du Cid ou d'Horace s'inscrirait dans la continuité du genre des tragédies romaines. Et Lancaster, distinguant dans l'œuvre de Campistron les tragédies héroïques et patriotiques de celles qui sont davantage psychologiques et pathétiques, choisit de classer Virginie dans le premier groupe, oubliant la place éminente accordée dans ce texte aux larmes et à la sensibilité.

En effet, il ne suffit pas de situer l'action de sa pièce à Rome pour écrire une tragédie romaine. Tout en s'inspirant de l'œuvre de Tite-Live, Campistron infléchit considérablement le propos de l'historien et nous livre avec sa Virginie une tragédie consacrée tout autant à la sphère intime et domestique qu'aux péripéties politiques. Par les transformations qu'elle apporte aux modèles anthropologiques et littéraires antérieurs, par la place qu'elle assigne au sentiment et au motif des larmes, qui ne sauraient se réduire au rang d'ornementations lyriques mais apparaissent comme des éléments dramaturgiques essentiels, la première pièce de Campistron traduit un infléchissement notable de la tragédie qui signale l'originalité du poète et, par bien des aspects, annonce le théâtre pathétique du siècle à venir. Nous tenterons de mettre en lumière cette originalité en montrant que le primat accordé à la sensibilité se traduit à la fois dans la caractérisation des différents

${ }^{1}$ Frères Parfaict, Histoire du théatre français, Paris, 1747, p. 363.

${ }^{2}$ La Porte, «Théâtre de Campistron », L'Observateur littéraire, Amsterdam, 1759, t. III, p. 218.

3 D’Alembert, «Éloge de Campistron », CEuvres, t. II, Paris, Belin, 1821, p. 571. 
personnages du drame et dans la définition d'un nouveau type d'héroïsme, puis nous nous interrogerons sur les prolongements politiques de cette éthique sensible.

Dans la préface de sa tragédie, Campistron rend hommage à Tite-Live qui lui a fourni le sujet de Virginie au livre III de son Histoire romaine. À cette source explicite s'ajoute, selon Dorothy F. Jones la Virginie romaine de Michel Le Clerc, tragédie en cinq actes publiée en $1645^{4}$. Chez ces deux auteurs, Campistron puise une inspiration indéniable. Toutefois, il s'éloigne considérablement de ces textes dans le traitement qu'il propose de cette anecdote historique. Tite-Live relate en effet l'enlèvement, par Appius, de Virginie, fille du centurion Verginius et fiancée du tribun Icilius à seule fin d'illustrer la corruption et le caractère tyrannique du pouvoir des décemvirs. Mettant l'accent sur la querelle judiciaire qui oppose les défenseurs de la jeune fille aux représentants du pouvoir politique plutôt que sur le calvaire de l'héroïne -à qui il ne donne d'ailleurs jamais la parole- l'historien entend éclairer son lecteur sur les causes de la chute du régime des décemvirs. Aussi son propos est-il essentiellement politique et le geste de Verginius qui poignarde sa fille afin de préserver son honneur semble davantage fournir le prétexte à une révolte populaire et à un changement de régime que constituer le dénouement d'une tragédie intime. L'académicien Michel Le Clerc perçoit pourtant, en 1645, les ressources tragiques de cet infanticide exemplaire et en fait le dénouement d'une tragédie politique significativement intitulée La Virginie romaine. Tout en accroissant le pathétique de la situation en développant l'amour qui unit le couple d'amants constitué par Virginie et Icile et les liens affectifs qui relient la jeune fille à son père, Le Clerc s'attache avant tout à peindre l'héroïsme de ses personnages, prêts à se sacrifier pour la liberté de Rome et à périr au nom de l'honneur. Aussi ne pleure-t-on guère dans cette tragédie où le stoïcisme et la fermeté d'âme semblent primer l'expression de la sensibilité ${ }^{5}$. Celle-ci est même explicitement condamnée par les personnages. Virginie s'exclame à la scène 1 de l'acte III, s'adressant à ses yeux :

Versez, versez du sang aussitôt que des pleurs,

Et craignez qu'aujourd'hui ma main ne vous arrache

\footnotetext{
${ }^{4}$ Dorothy F. Jones, Jean de Campistron : a study of his life and work, University of Mississippi, Romance monographs, 32, 1979, p. 19.

5 Cette préférence accordée à la dimension politique par rapport à la tragédie intime se retrouvera, près d'un siècle et demi plus tard, dans la Virginie de La Harpe (1786) qui s'inscrit délibérément dans la veine des tragédies romaines. Symptomatique du refus de céder à la vogue du pathétique et de la réticence à verser dans le tragique bourgeois est la scène 4 de l'acte I où le personnage de Plautie (hérité de Campistron mais considérablement défiguré par La Harpe) rappelle à Virginie ses devoirs de romaine. Alors que celle-ci est sur le point d'épouser Icilius, elle lui déclare : «Prends garde qu'aux autels portant un juste hommage/ D’un si doux avenir la trop flatteuse image/ Te fasse oublier Rome en présence des dieux./ Qu'ils entendent ce nom mêlé dans tous vos vœux./ Ah! Quand votre union sous leurs yeux se consomme/ Priezles de finir l'esclavage de Rome. / Vous aimez la patrie, et ce grand sentiment/ Jamais d'un cœur romain ne s'éloigne un instant».
} 
Si vous n'en répandez pour laver cette tâche. ${ }^{6}$

L'opposition des larmes et du sang est révélatrice des devoirs attachés à la condition de romaine qui implique une absolue fermeté devant l'ennemi. Agir plutôt que pleurer, offrir à l'adversité le visage impassible de l'honneur invaincu, maitriser les élans de l'humanité pour atteindre au sublime détachement de soi, ne jamais accepter sa position de victime, voilà ce qui fait de Virginie le digne pendant des héros cornéliens. La jeune fille est pourtant sensible et s'écrie, à la scène 3 de l'acte III, après avoir appris la condamnation de son amant :

Éclatez mes douleurs, il n'est plus temps de feindre. ${ }^{7}$

Mais, conformément à l'éthique romaine, cet épanchement sensible est bien vite réprimé par Virginius, qui rappelle sa fille à son devoir :
VIRGINIUS :
Regarde d'un œil sec creuser ma sépulture.
Tes larmes corrompraient la pureté du sang.
VIRGINIE :
Ah ! Mon père, pardon si j'ai pu consentir
Aux tendresses du sang qu'on ne peut démentir.
J'ai cru que je pouvais paraitre un peu sensible
Sans trahir l'intérêt d'un honneur invincible
Et que de mille assauts on verra triompher.
Ma douleur vous déplait, je la veux étouffer.
S’il faut que vous mouriez, m’y voilà résolue.
Ma faiblesse, tais-toi, te voilà convaincue. ${ }^{8}$

Pour Le Clerc, les larmes portent atteinte à la dignité du héros romain, elles l'abaissent au rang de l'humanité commune et atténuent ce que sa conduite peut avoir de sublime. La sphère politique et la pensée de l'honneur priment à tel point les liens individuels que ce n'est qu'après avoir sacrifié sa fille que Virginius pourra, au dénouement, mêler «au sang qu'elle versa un déluge de larmes ».?

La situation est très différente chez Campistron qui, s'il s'inspire des textes de Tite-Live et de Le Clerc, y apporte un certain nombre de modifications. Il recentre tout d'abord l'action sur le sort de Virginie. Il n'est plus question chez lui, comme dans La Virginie romaine, d'une tentative d'assassinat orchestrée par Icile contre Appius et l'on ne trouve pas trace du débat judiciaire portant sur des questions de procédure, qui occupe l'essentiel des pages consacrées par Tite-Live à l'aventure de Virginie. Le nom de Rome

\footnotetext{
${ }^{6}$ Le Clerc, La Virginie romaine, Paris, Toussaint Quinet, 1649, acte III, scène 1, p. 26.

${ }^{7}$ Le Clerc, La Virginie romaine, acte III, scène 3, p. 35.

${ }^{8}$ Ibid., acte III, scène 4, p. 37-39.

9 Ibid., acte V, scène 2, p. 61.
} 
n'est guère prononcé dans la pièce et la révolte finale semble suscitée davantage par l'injustice dont est victime l'héroïne que par l'insatisfaction politique du peuple. Le signe le plus éloquent de ce relatif retrait des préoccupations politiques est l'absence de Virginius qui, conformément à la chronique livienne, mais à rebours de ce que l'on peut observer chez Le Clerc, est absent de Rome durant les événements et ne revient qu'au dénouement. Absent de la scène, puisque son retour ne fait l'objet que d'un récit, Virginius est en outre chez Campistron singulièrement absent des discours des autres personnages. L'éviction du père est hautement symbolique et révélatrice de l'infléchissement imposé à la fable originelle par Campistron. Bannissant Virginius, allant jusqu'à lui confisquer son rôle historique en transformant l'infanticide final en suicide de Virginie, Campistron prend ses distances vis-àvis de l'histoire et de la politique. Ce parti pris apparait d'autant plus nettement que le dramaturge profite du vide créé par l'absence de Virginius pour inventer de toutes pièces le personnage de Plautie, mère de l'héroïne qui nous est présentée dans les premiers actes comme constamment éplorée ${ }^{10}$. Par le biais de Plautie, Campistron réhabilite la sphère privée et accrôit le rôle dévolu aux personnages féminins. Toutes les conditions semblent donc réunies pour placer cette tragédie sous le signe de la sensibilité Cette évolution est également sensible dans la manière dont Campistron traite la calomnie qui sert de prétexte à la persécution de Virginie. Tite-Live en effet relate qu'Appius, pour pouvoir s'emparer de la jeune fille qu'il convoitait, avait fait courir le bruit que Virginie n'était pas la fille de Virginius mais celle d'un esclave appartenant à l'un de ses clients, et qu'il la réclamait à ce titre. Cette accusation, très vite reconnue comme mensongère, est presque immédiatement évacuée par les textes de Tite-Live et de Le Clerc, mais Campistron s'y arrête et entreprend d'en dénoncer la fausseté en fondant sa démonstration, non plus sur l'ethos de l'accusateur, mais sur les qualités de la victime. Si Icile, arguant de l'héroïsme et de la grandeur d'âme de Virginie pour justifier son extraction aristocratique, use d'un argument classique ${ }^{11}$, Plautie, quant à elle, recourt à une justification

\footnotetext{
10 Son apparition sur scène est précédée par une réplique de Fabien qui annonce à Appius l'arrivée de Plautie « aux pleurs abandonnée », laissant échapper de «fréquents soupirs » (Acte I, scène 2). Prévenu, Appius congédie Claudius, en lui disant : «Vous, d'une mère en pleurs, évitez les transports », réplique prémonitoire qui annonce, d'une certaine manière, le dénouement et la méfiance suscitée chez les méchants par les pouvoirs des larmes. Plautie ellemême, dès son entrée en scène, se présente sous les traits d'une suppliante s'exclamant: «Ah! Seigneur, écoutez les douleurs d'une mère », avant d'exprimer ses doléances dans une réplique éminemment pathétique : «En vain depuis deux jours errante dans ces lieux/ Les pleurs que j'ai versés ont épuisé les yeux./ En vain de tous côtés mes cris se font entendre:/ De son destin encor je n'ai pu rien apprendre/ Et je trouve partout, dans mes soins empressés/ Des gardes interdits, des visages glacés/ Qui redoutent ma vue et prêts à se confondre/ Se dérobent à moi, sans daigner me répondre. » (acte I, scène 3, p. 16-17).

11 Témoin de la grandeur d'âme de l'héroïne, il s'exclame à la scène 2 de l'acte II (p. 34) : «Que ce noble discours pleinement justifie/ Le véritable sang dont vous êtes sortie./ Un cœur dans l'esclavage et d'un vil sang formé/ D'un courage si grand n'est jamais animé./ Et quelque fier qu'il soit, toujours quelque faiblesse/ Découvre tôt ou tard sa première bassesse ». La fermeté toute romaine dont fait preuve Virginie suffit à justifier son ascendance illustre. Cependant,
} 
plus originale, qui témoigne de la prégnance d'une éthique de la sensibilité. Elle s'écrie ainsi à la scène 3 de l'acte I :

Non, non, elle est ma fille et j'en crois mon amour ;

Mon cœur frémit, mon sang s'émeut de cette injure.

Je sens trop fortement s'expliquer la nature

Et je cède à la voix de ces instincts secrets

Qui parlant à nos cœurs ne les trompent jamais. ${ }^{12}$

Évoquant cette voix du sang ou voix de la nature qui connaitra une fortune considérable au dix-huitième siècle, elle souligne la distance anthropologique qui sépare le modèle éthique représenté par la tragédie de Le Clerc de la nouvelle idéologie sensible, à l'œuvre chez Campistron. La nature, domptée et refoulée chez Le Clerc, ressurgit chez Campistron libérée de la condamnation morale et du soupçon de bassesse qui pesaient sur elle. Tout se passe chez Campistron comme si sensibilité et hérö̈sme n'étaient plus incompatibles et l'on assiste à une remise en question du modèle romain, particulièrement évidente dans le traitement des personnages principaux.

La place accordée au couple formé par Icile et Virginie est éminemment représentative de cette évolution. Les types de la tragédie romaine se voient en effets investis par la sensibilité et s'en trouvent considérablement modifiés. C'est le cas d'Icile, fier tribun chez Tite-Live, transformé chez Campistron en parfait amant, plaçant sur le même plan ses devoirs privés et publics. Alors que l'historien romain le dépeignait en politique soucieux de sa réputation, défendant en Virginie bien moins l'objet de sa tendresse que son propre honneur et le droit des citoyens ${ }^{13}$, dans la tragédie de Campistron, les motivations intimes et l'amour semblent primer sur les préoccupations politiques et sur le souci du rang. Ainsi Icile passe-t-il plus de temps en entretiens avec Virginie qu'à agir sur la scène pour la chute des

Icile, comme on le verra par la suite, est bien loin d'affirmer ici que la noblesse morale est incompatible avec l'humilité de la naissance.

12 Campistron, acte I, scène 3, p. 19.

13 C'est en ces termes qu'il s'adresse à Appius sur le forum : "C'est par les armes qu'il faudra me faire partir d'ici, Appius, si tu veux que le silence se fasse sur ce que tu veux garder secret. J'ai l'intention d'épouser cette jeune fille et je l'épouserai vierge. Tu peux bien faire venir tous tes licteurs et ceux de tes collègues, ordonner qu'on prépare les verges et les haches : la fiancée d'Icilius ne quittera pas la maison de son père. N'allez pas croire parce que vous nous avez privés de l'assistance des tribuns et de l'appel au peuple, ces deux bastions de la liberté, que nous laisserons nos femmes et nos enfants subir la tyrannie de vos caprices. Passez votre colère sur notre dos, sur notre nuque : sauvons du moins la pudeur. Si vous portez la main sur cette jeune fille, je demanderai l'aide des citoyens ici présents pour ma fiancée, Verginius celle de ses soldats pour sa fille; nous tous, nous implorerons le secours des dieux et des hommes et il faudra nous massacrer avant d'exécuter ta décision» (Tite-Live, Histoire romaine, GF Flammarion, t. I, livre III, 45, p. 333-334). On le constate aisément : il n'est guère question de la pauvre Virginie dans ce discours de romain défendant son honneur bafoué. Icile parle pour lui et semble ne voir dans la situation de sa fiancée qu'un affront personnel et une illustration de la corruption de la pratique politique des décemvirs. Bien différente sera la position de l'Icile de Campistron, qui réagit avant tout en amant. 
décemvirs $^{14}$, ce qui fait dire aux frères Parfaict qu’il «pleure, gémit, se donne beaucoup de mouvements sans rien opérer $»^{15}$. Cette allégeance aux codes de la sensibilité explique également le fait qu'il ne se laisse pas émouvoir par les calomnies d'Appius et qu'il se montre constant dans son amour, au grand étonnement de Sévère qui, dès la scène 1 de l'acte II, souligne le caractère atypique du comportement d'Icile qui déroge à l'éthique romaine :

Seigneur, est-ce un effet de la seule pitié

Ou le simple devoir d'un reste d'amitié?

Car je ne pense pas, dans sa misère extrême,

Averti de son sort par Plautie elle-même

Quand le sort l'abandonne au plus cruel malheur

Que vous sentiez pour elle une honteuse ardeur.

Non, je ne croirai point qu'un aussi grand courage

Puisse avilir ses vœux jusque dans l'esclavage,

Qu'Icile jusque-là pût jamais s'abaisser. ${ }^{16}$

À ce rappel de son statut et de ses devoirs, Icile répond en amant constant et en homme délivré des préjugés de rang:

Mais quand les dieux auraient fait naitre Virginie

Dans la honte des fers et dans un rang plus bas

Quel que fût son destin, je ne changerais pas.

Plus on veut l'abaisser, plus je sens que je l'aime,

Si ses malheurs sont grands, mon amour est extrême.

[...] Mais du moins aujourd'hui mon amour seul éclate

Et mon ambition, n'ayant rien qui la flatte,

Je ferai hautement triompher en ce jour

La générosité, la constance et l'amour. ${ }^{17}$

Les propos d'Icile frappent par leur modernité. Affichant ouvertement la préférence pour les devoirs et les attachements privés au détriment des conventions sociales, rejetant les valeurs aristocratiques du rang et de la gloire

${ }^{14}$ Ce qui frappe dans le personnage d'Icile tel que le dépeint Campistron, c'est sa tendance à se complaire dans la déploration et dans la tendresse. Éloquent est, à cet égard, le début de la scène 4 de l'acte IV qui se démarque considérablement de la dernière entrevue de Virginie et de son père chez Le Clerc. Loin de répudier toute marque de sensibilité, l'Icile de Campistron en loue au contraire les délices : «Madame, qu'il m'est doux de vous parler encore,

/ De pouvoir attendrir la beauté que j'adore,/ Et de voir une fois au moins avant ma mort/ Vos yeux donner des pleurs à mon funeste sort ».

${ }^{15}$ Frères Parfaict, op. cit., p. 365.

${ }_{16}$ Campistron, acte II, scène 1, p. 27.

17 Campistron, acte II, scène 1, p. 28. Icile ajoute : «S'il est des cœurs mal faits et d'indignes amants/ Qui suivent dans leurs vœux ces lâches sentiments,/ Pour moi, n'en doute point, quand j'aime Virginie,/ C'est à d'autres objets que mon cœur sacrifie,/ Les grandeurs que le sort peut ravir en un jour/ N'ont jamais attiré mes vœux ni mon amour./ La fermeté d'esprit, la grandeur de courage,/ La pureté du cœur, voilà ce qui m’engage./ Ce qui dépend du sort est pour moi sans appas/Et j'aime les vertus qui n'en dépendent pas ». 
pour les valeurs chrétiennes que sont la constance et la communion empathique, Icile apparait davantage comme un philosophe des Lumières que comme un héros cornélien. Il préfigure, dans une certaine mesure, le comte de la Nanine de Voltaire qui, lui aussi, affirme préférer les vertus naturelles au prestige de la fortune et de la naissance ${ }^{18}$. Se fait jour dans ses propos une forme d'hérö̈sme originale, compatible avec l'acceptation du sort et l'humilité. Les malheurs qui frappent Virginie offrent à Icile l'occasion de manifester la profondeur et la pureté de ses sentiments et, à ce titre, ils apportent la preuve de la générosité du héros, tout en fournissant l'occasion d'une révélation des vraies valeurs, celles d'une sensibilité qui se voit préférée à l'orgueil. Le caractère hétérodoxe du comportement d'Icile frappe Virginie qui, à la scène 2 de l'acte II, interrompt les serments amoureux de celui-ci, en objectant :

Ah ! Seigneur, quelle erreur est la vôtre.

Lorsque vous me verrez dans un rang odieux...

Mais Icile ne se laisse pas démonter et persiste dans sa profession de foi sensible, allant jusqu'à inverser les principes de l'éthique aristocratique. Il répond :

J'aurai le même cœur, j’aurai les mêmes yeux.

Vous conserverez tout ce que mon cœur adore,

Vous aurez vos vertus et vous aurez encore

Pour m'attacher à vous par un lien plus fort

Vos craintes, vos douleurs, les injures du sort.

Oui, pour serrer les nœuds d'une chaîne si belle

Vos disgrâces auront une force nouvelle. ${ }^{19}$

On assiste ici à l'émergence d'une sorte d'évangile sensible qui vient se substituer au modèle hérö̈que cornélien et instaure un type de sociabilité radicalement autre. Dans cette célébration de la sensibilité et de la compassion, on peut également lire l'émergence d'une érotique proprement pathétique. La sensibilité semble régner en maittre sur Icile qui, tout entier absorbé par les

18 Celui-ci tient, en 1749, un discours très semblable à celui d' Icile. Épris d'une roturière, il défend, face à la baronne les droits de la nature et de l'humanité face aux préjugés aristocratiques. Il déclare ainsi, à la scène 1 de l'acte $\mathrm{I}:$ « Je ne prends pas, quoi qu'on en puisse croire/ La vanité pour l'honneur et la gloire./ L'éclat vous plait; vous mettez la grandeur/ Dans des blasons; je la veux dans le cour ». Il est intéressant d'observer que, de même que Virginie incarne, face à Icile, la fidélité à l'héroïsme traditionnel, Nanine, dans la pièce de Voltaire demeure, à la différence de son amant, imprégnée de l’idéologie commune. Lisant, à la scène 5 de l'acte I « un livre anglais » dont on lui a fait présent, elle énonce un point de vue qui semble bien timoré après la profession de foi philosophique et égalitariste du comte. Elle dit en effet: «L'auteur prétend que les hommes sont frères,/ Nés tous égaux; mais ce sont des chimères ;/ Je ne puis croire à cette égalité ». Dans les deux œuvres, le relatif conservatisme des héroïnes n'a d'autre fonction que de mettre en évidence leur valeur et leur vertu, et de les faire ainsi aimer d'un public, sensible, chez Voltaire, à l'humilité de la jeune fille et, chez Campistron, à l'injustice faite à Virginie.

${ }^{19}$ Campistron, acte II, scène 2, p. 33. 
charmes de la tendresse, en oublierait presque ses devoirs de romain. C'est paradoxalement Virginie qui lui remet en mémoire la hiérarchie des valeurs traditionnellement en usage dans la tragédie ${ }^{20}$. Annonçant à Icile, à la scène 4 de l'acte IV, qu'elle a décidé de mourir pour éviter le déshonneur, elle reproche à son amant de verser des larmes sur son sort et s'écrie : "Vous tremblez et vous êtes romain». Loin d'en rougir, comme l'auraient fait les héros de Le Clerc, Icile lui répond aussitôt :

Oui, je tremble sans doute, et je vous le confesse :

Mais mon cœur s'applaudit d'avoir cette faiblesse. ${ }^{21}$

Revendiquant la valeur de l'émotion, attentif à la voix du sentiment, Icile est porteur d'une nouvelle éthique hérö̈que, tournée non plus exclusivement vers l'action éclatante et le sublime politique mais proclamant les droits de l'humanité et de la compassion ${ }^{22}$.

20 Annonçant à son amant que leur union est rendue impossible par les révélations concernant sa naissance, elle lui promet, dans un discours qui rappelle celui que tient, dans l'Iphigénie de Racine, l'héroïne à Achille à la scène 2 de l'acte $\mathrm{V}$, des consolations militaires : "Mais l'honneur immortel qu'au milieu des combats/ Votre rare valeur promet à votre bras,/ Le généreux désir de servir sa patrie/ Pourront de votre esprit effacer Virginie./ Ou, si ces nobles soins ne peuvent l'en bannir,/ Pour en combattre au moins le triste souvenir/ Vous pourrez opposer après votre victoire/ Aux chagrins de l'amour, les plaisirs de la gloire ». (acte II, scène 2, p. 33). Virginie semble donc, plus que son amant, imprégnée de l'idéal héroïque romain.

21 Campistron, acte IV, scène 4, p. 78.

${ }^{22}$ Face à ce héros sensible, c'est Virginie qui semble la plus digne héritière du modèle hérö̈que, à tel point que La Porte se prend à imaginer une tragédie parfaite où Icile «n'aurait cédé à sa maîtresse ni en grandeur d'âme ni en délicatesse de sentiment» et où "les scènes où ils se trouvent ensemble auraient eu tout l'effet qu'on devait attendre naturellement et de deux amants et de deux romains » (op. cit., p. 218). Comme toute bonne héroïne cornélienne, Virginie semble en effet d'autant plus consciente des devoirs dus à son honneur et à son rang qu'elle s'en voit privée par les accusations d'Appius et de Claudius. Alors qu'Icile ne cesse d'invoquer les droits et les devoirs du cœur, la jeune fille ne cède qu'à ceux du sang, trouvant dans l'allégeance au modèle aristocratique une consolation à ses malheurs présents et une preuve de leur injustice. Elle le dit à Plautie à la scène 4 de l'acte I : «Le sang a dans mon sein transmis votre courage:/ Attentive aux leçons qu'ont tracées mes aïeux/ Leur exemple sans cesse est présent à mes yeux ;/ De mes jours malheureux, je finirai la course/ Sans qu'aucune faiblesse en ternisse la source./ Le plus cruel trépas me semblera trop doux/ Mourant avec le nom que j'ai reçu de vous » (acte I, scène 4, p. 22). Consciente de sa valeur, Virginie est proche de la figuration traditionnelle des héroïnes romaines et conserve bien des traits de l'héroïne de Le Clerc. Aussi n'est-ce pas un hasard si c'est elle qui se voit attribuer dans le texte de Campistron les quelques citations cornéliennes qui s'y trouvent. Reprenant un vers d'Horace, elle dit à Icile à la scène 2 de l'acte II : «Ma générosité doit seconder la vôtre » (p. 34 et Horace, v. 437) et, c'est encore Horace qu'elle cite à la scène 4 de l'acte IV, lorsqu'à son amant qui lui demandait: "Qu'avez-vous résolu, Madame ?», elle répond : «De mourir» (p. 78, Horace, v. 1021). Virginie semble donc dépositaire de la conception traditionnelle de l'héroïsme romain, ce que vient d'ailleurs accréditer le dénouement original infligé à sa tragédie par Campistron. Alors que chez Tite-Live, c'est Virginius qui poignardait sa fille afin de la sauver du déshonneur, chez Campistron, les choses se passent très différemment. Chargé du récit, Camille raconte : «De sa constante fille il [Virginius] veut percer le cœur,/ Mais en vain pour ce coup son courage s'apprête,/ Quand il croit l'achever sa tendresse l'arrête:/ Car, à peine a-t-il vu le couteau près 
Non contentes de déterminer de nouveaux modes comportementaux et d'infléchir la conception traditionnelle de l'héroïsme, les pratiques lacrymales jouent par ailleurs un rôle essentiel dans la perception du schéma actanciel et la polarisation morale des différents personnages. C'est ce que l'on observe à la scène 2 de l'acte $V$, où Virginie compare deux types de rapport aux larmes, attachés respectivement à chacune des parties en conflit dans la fable tragique. S'adressant à Plautie qui pleure leur séparation prochaine, elle déclare :

Je conçois par les pleurs dont votre amour m'honore

Quelle vive douleur, quel chagrin vous dévore

Et je ne vois que trop qu'une tendre pitié

Vous fait de tous mes maux ressentir la moitié.

Elle constate ainsi le fonctionnement normal de la compassion, auquel va bientôt s'opposer un autre type de réception des larmes, manifestant, cette fois, un détournement pervers de la sensibilité. Virginie ajoute en effet:

Cependant, retenez vos soupirs et vos larmes ;

Au fond de votre cœur renfermez vos alarmes.

Claudius va venir, faites un noble effort;

De tous vos déplaisirs modérez les transports.

Nos regrets, les ennuis où nous sommes en proie

D'un cruel ennemi redoubleraient la joie.

Ne permettez donc pas que ses barbares yeux

Jouissent des douleurs de nos derniers adieux..$^{23}$

du sein,/ Que la nature semble avoir glacé sa main./ Il demeure immobile à ce triste spectacle./ On court. À son dessein chacun veut mettre obstacle./ Virginie en tremblant voit venir ce secours,/ Qui hasarde sa gloire en conservant ses jours./ Elle se hâte alors de terminer sa vie,/ S'élance sur le fer et d'une main hardie/ Prend celle de son père et poussant le couteau/ S'en frappe, tombe et s'ouvre un chemin au tombeau » (acte V, scène 7, p. 100). Alors que le héros de l'histoire se voit réduit à l'impuissance et paré d'une sensibilité inédite, c'est paradoxalement la victime qui, sacrifiant tout à son honneur, agit et accomplit le geste sublime. La situation de la tragédie de Le Clerc se trouve donc inversée. Pour être de nature aristocratique, l'héroïsme de Virginie n'en est pas moins un héroïsme passif, un héroïsme de l'acceptation du sort et du sacrifice, comme en témoigne cet aveu à Camille, à la scène 5 de l'acte I : «N'importe en ce moment, quoi que le ciel ordonne/ À ses ordres sacrés mon âme s'abandonne ;/ Je respecte les traits qui partent de sa main/ Et je vais sans murmure attendre mon destin » (p. 25). En ce sens, autant sinon plus qu'aux hérö̈nes cornéliennes, Virginie est redevable à l'Iphigénie de Racine, héroïne fondamentalement pathétique et, comme elle, victime innocente. Avec Virginie, Campistron applique la leçon de Racine et inaugure un schéma que l'on retrouvera dans la majorité de ses tragédies. Cherchant à caractériser la dramaturgie de Campistron, Lanson déclare en effet que "pour toucher comme Racine, il quitte la voie de Racine» et «concentre l'intérêt sur la victime qui redevient le personnage principal » (Gustave Lanson, Esquisse d'une histoire de la tragédie française ,Paris, Champion, 1927, p. 129). Par ce biais, se met en place un héroïsme foncièrement pathétique, suscitant les larmes autant que l'admiration. Ce modèle deviendra dominant dans le théâtre du XVIIIe siècle.

${ }^{23}$ Campistron, acte V, scène 2, p. 89. 
Il y aurait donc deux réactions possibles au spectacle des larmes: l'une vertueuse, celle de Virginie qui sent ses douleurs atténuées par les marques de sympathie et la «tendre pitié » que lui témoigne sa mère, et l'autre vicieuse, celle de Claudius, à qui les larmes de ses victimes procurent une jouissance perverse. Le contre-modèle du héros sensible ne serait donc pas tant le héros cornélien ou romain qui s'applique à dompter la nature que les personnages foncièrement insensibles, sourds à l'humanité la plus élémentaire et inaccessibles à la compassion. Cette opposition axiologique de l'âme tendre et du cœur froid va devenir un lieu commun de la littérature du XVIII ${ }^{\mathrm{e}}$ siècle, que ce soit dans le théâtre des Philosophes ou dans les romans de Sade.

Le méchant est donc celui qui ne pleure pas et, plus généralement, celui qui refuse de souscrire aux lois de la sensibilité. Cette description correspond parfaitement au personnage d'Appius, qui use des larmes comme d'un masque afin de mieux manipuler les héros et ainsi parvenir à ses fins. Mais, s'il n'est pas sensible, Appius est passionné, ce qui en fait, selon Dorothy Jones, un parent du Néron de Britannicus ${ }^{24}$ et donc un héros dans la veine racinienne. Alors que la sensibilité, sous la forme dépassionnée de la compassion et de la tendresse, est explicitement valorisée par le texte et les personnages principaux, Campistron nous présente l'amour qu'Appius porte à Virginie comme une maladie. Comme Phèdre, Appius tait son mal et cache cet «injuste chagrin qui [le] trouble et [le] gêne $»^{25}$. Il ne s'en ouvre qu'à Claudius, lui avouant sa passion à la scène 1 de l'acte $\mathrm{I}$, dans un propos qui trahit à la fois les motivations du personnage et la condamnation éthique attachée à la passion amoureuse, condamnation intégrée par Appius lui-même qui explique :

Je me suis tu longtemps, et peux me taire encore,

Loin de faire éclater ce feu qui me dévore,

Je dois plus que jamais le cacher en ce jour. ${ }^{26}$

Passion honteuse et nocive, l'amour est conçu par le méchant même comme un dérèglement qui porte au mal et qui, à la différence de la sensibilité, ne saurait se montrer. Cette passion illégitime ne peut en effet se satisfaire qu'en recourant à la dissimulation et à la manipulation d'autrui. Aussi Appius met-il au point une stratégie perverse, fondée sur l'hypocrisie et l'instrumentalisation de la sensibilité.

Déguisons ce forfait, couvrons-en la noirceur

\footnotetext{
24 Op. cit., p. 28.

25 Campistron, acte I, scène 1, p. 8. Cet amour, existant chez Tite-Live et chez Le Clerc était toutefois relativement mis en sourdine dans leurs œuvres. Alors que l'Appius de La Virginie romaine évoquait sa flamme en des termes pré-raciniens ("Je me plais à nourrir un serpent qui me tue », acte I, scène 1, p. 5), Virginius demeurait sceptique et préférait considérer la passion d'Appius comme un prétexte mensonger à la persécution de Virginie (" Je sais que pour ternir l'éclat de ma famille/Tu feins depuis longtemps d'être épris de ma fille », acte II, scène 2, p. 16).

${ }^{26}$ Campistron, acte I, scène 1, p. 12.
} 
Et faisons admirer ce qui ferait horreur.

Si la vertu souvent passe pour imposture

Le crime imite aussi la vertu la plus pure

Et mon coupable amour sera mieux écouté

Sous un prétexte adroit de générosité.

[...] Sous ces dehors flatteurs, je cacherai mon crime,

Par-là je gagnerai son cœur et son estime.

Et l'on imputera par ce subtil détour

À la seule pitié les effets de l'amour. ${ }^{27}$

L'hypocrisie d'Appius traduit donc à la fois la prégnance de la nouvelle norme éthique que constitue le modèle sensible, et le détournement pervers de celleci. En ce sens, Appius apparait comme un véritable contre-modèle, opposant à la profondeur des sentiments naturels de pitié ou de générosité la tartufferie de mœurs et transformant les élans altruistes d'une compassion synonyme d'oubli de soi en feinte destinée à servir ses intérêts personnels. Réduite à une posture mensongère et à des discours creux, la sensibilité affichée par Appius n'est qu'un moyen de parvenir à ses fins. Aussi l'abandonne-t-il dès lors que, repoussé par Virginie, il constate l'inefficacité de sa stratégie ${ }^{28}$.

Il met ainsi un terme aux innombrables protestations de foi sensibles prodiguées à Virginie et à Plautie au cours des trois premiers actes. En parfait pharisien, Appius use de la sensibilité comme d'un masque rhétorique, se forgeant à peu de frais l'ethos d'un magistrat aussi équitable que compatissant. Mais cette sensibilité affichée se limite à des consolations verbales, Appius ne faisant jamais suivre d'effet ses protestations de sympathie, ainsi que le montre la scène 3 de l'acte I. À la mère éplorée qui vient lui demander de l'aide, il répond en effet :

Ne doutez point, Madame,

Que je ne sois frappé du trouble de votre âme.

J'ai craint avec raison de vous voir en ces lieux

Et que votre douleur n'éclatât à mes yeux.

J'ai fait plus, j'ai tâché longtemps de me défendre

De causer tant de pleurs que je vous vois répandre,

Mais mon cruel devoir, le plus fort dans mon cœur

D’une pitié craintive est demeuré vainqueur,

J'ai cédé, j’ai suivi la sévère justice.

Enfin, que vouliez-vous, Madame, que je fisse?

Chargé par tout l'État du pouvoir souverain...

Il est alors interrompu par Plautie, qui rétorque :

${ }^{27}$ Campistron, acte I, scène 1, p. 13.

${ }^{28}$ C'est ainsi qu'il s'écrie à la scène 6 de l'acte IV : «C'en est fait. Bannissons la pitié de mon âme » (p. 82). 
Osez-vous vous parer d'un prétexte si vain ?29

Cet extrait illustre parfaitement l'hétérogénéité radicale des univers éthiques incarnés par ces deux personnages, ainsi que le renversement des valeurs qui s'est opéré dans le passage de la Virginie romaine de Le Clerc à la Virginie de Campistron : Plautie récuse en effet la distinction et la hiérarchie établies par Appius entre devoirs publics et devoirs vis-à-vis de l'humanité souffrante. À ses yeux, l'ordre du cœur et celui de la cité ne sauraient entrer en concurrence et la responsabilité publique ne permet en aucun cas de cautionner une injustice morale. La position adoptée par Appius est, aux yeux de Plautie, la marque d'une disconvenance aussi bien éthique que politique qui, pour le spectateur omniscient, trouve son explication dans l'imposture d'Appius, puisqu'en effet ni le droit ni la nature n'autorisent en réalité les actions de ce dernier. Remettant en cause l'impassibilité et l'impartialité des juges, et dénonçant l'acceptation par Appius d'une situation injuste, Plautie se fait le porte-parole d'une vision du monde optimiste fondée sur la foi en une réconciliation de l'ordre naturel et d'un ordre social gouverné par la sensibilité. Elle énonce ainsi une croyance qui sera l'un des thèmes récurrents du théâtre des Philosophes au XVIII ${ }^{\mathrm{e}}$ siècle $^{30}$. L'argument invoqué par Appius pour justifier sa passivité et expliquer son acceptation de la situation ne peut donc la convaincre et ne fait que l'amener à soupçonner l'imposture du décemvir, à la fois sur le plan moral et sur le plan politique.

L'originalité de Campistron est en effet de faire des larmes un enjeu et une ligne de partage axiologique non seulement dans le domaine éthique mais aussi dans celui de la politique - deux domaines qui désormais ne sont plus étanches - et d'opposer, à travers les personnages d'Appius et de Plautie, deux types de pratiques du pouvoir tout autant que deux visions du monde. Avec le personnage de Plautie, les larmes vont en effet venir

${ }^{29}$ Campistron, acte I, scène 3, p. 16-17. Cette idée revient plusieurs fois dans le discours d'Appius qui s'exclame encore, à la scène 3 de l'acte I : "Inexorable loi d'un devoir trop sévère!/ Qui nous fait bien souvent condamner à regret/ Ceux pour qui notre cœur se déclare en secret» (p. 20). Et, à la scène 4 de l'acte II : «Hélas! N'en doutez point, votre disgrâce extrême/ Plus que vous ne pensez me déchire moi-même. / Et pour porter mon âme à finir vos malheurs/ Vous n'avez pas besoin du secours de vos pleurs./ Votre seule jeunesse et les soins d'une mère/ À qui mille raisons vous ont rendue si chère,/ D'un père si fameux les illustres exploits/ Lorsqu'ils parlent pour vous ont de pressantes voix./ Souvent par ces égards mon âme s'est émue,/ De vous rendre à leurs cris, elle était résolue,/ Si l'austère devoir d'un emploi glorieux/ Cette droite équité prescrite par les dieux,/ Si la peur des remords qui suivent l'injustice/ M'eût permis de vous faire un si grand sacrifice,/ Et n'eût, malgré l'effort d'une tendre pitié/ Fait durer des malheurs dont je sens la moitié » (p. 38).

${ }^{30}$ Elle annonce aussi la transformation des figures de juges et de magistrats à venir dans le drame. Voir par exemple Le Juge (1774) et L'Indigent (1772) de Mercier qui présentent des représentants de l'autorité judiciaire une image idyllique, modelée sur les valeurs de la sensibilité et Jean Calas ou L'École des juges (1791) de Marie-Joseph Chénier qui se clôt sur un plaidoyer en faveur de l'humanité de la justice et propose un face à face de deux magistrats qui, par bien des aspects, rappelle la confrontation d'Appius et Plautie. 
contaminer le champ politique et imposer une conception nouvelle de l'ordre public.

Dans une perspective sentimentale, les représentants de l'autorité, garants de l'harmonie sociale, apparaissent comme les protecteurs naturels de l'innocence persécutée. Aussi Virginie et Plautie recourent-elles aux larmes afin de s'attirer le soutien des puissants. Partie prenante du débat judiciaire, les larmes deviennent ainsi arme politique. En témoigne cette proposition de Virginie à Camille, à la scène 3 de l'acte II :

Courons voir Appius, il peut nous secourir.

Que ses yeux soient témoins de mes vives alarmes;

Peut-être sera-t-il attendri par mes larmes. ${ }^{31}$

Dans l'éthique de la sensibilité qui imprègne la pièce, les larmes d'une femme opèrent théoriquement une séduction imparable, et les héroïnes, renouant avec l'origine persuasive du pathos, en usent comme d'un outil rhétorique. Or le pacte pathétique ne peut ici se réaliser faute d'une adhésion sincère des deux parties en présence aux valeurs qu'il implique. La supplique de Plautie, comme celle de Virginie, se heurte à l'insensibilité foncière d'Appius qui, s'il veut bien payer leur douleur de belles paroles, se borne à arborer le masque de la compassion et refuse de traduire en actes les sentiments qu'il prétend éprouver. L'attitude d'Appius trahit un dysfonctionnement de la sociabilité sentimentale et apparait comme une atteinte aux lois de l'humanité et aux devoirs du parfait monarque. L'impassibilité d'Appius est désignée comme anormale par Virginie qui s'exclame avec étonnement, à la scène 4 de l'acte II :

Perdrai-je tant de pleurs que vous voyez répandre?

Et n'obtiendrai-je point un utile secours

Qui des fers que je crains sauve mes tristes jours. ${ }^{32}$

Plautie elle aussi perçoit l'hypocrisie d'Appius et le prend à partie :

Faudra-t-il qu'à mes yeux on puisse reprocher

Qu'ils n'ont pas eu la force, hélas, de vous toucher?

Dans le temps qu'à vos yeux je suis presque mourante

Mon extrême douleur sera-t-elle impuissante?

D'un barbare projet vous connaissez l'auteur

Et mes tristes soupirs, mes transports, ma fureur

Mon désespoir mortel, mon ardente prière,

Tout vous prouve, seigneur, l'amitié d'une mère.

Faut-il d'autres raisons pour vous persuader?

Il en est mille encor à qui tout doit céder.

Considérez, seigneur... Mais mon âme troublée

Succombe à tant de maux dont elle est accablée,

31 Campistron, acte II, scène 3 , p. 37.

32 Campistron, acte II, scène 4, p. 37. 
Ma parole se perd... je cède à mes douleurs...

Hélas!... Je ne vous puis parler que par mes pleurs. ${ }^{33}$

Pressentant l'échec de son ambassade, Plautie, consciente de la faillite du discours articulé, quitte les figures convenues du pathos, abdique toute maitrise rhétorique et se laisse aller à l'expression la plus naturelle de la sensibilité qui, dès lors, apparaît bien moins comme persuasive que comme purement expressive. Dans cette gradation pathétique qui s'achève dans le silence des larmes, Plautie recourt au style "coupé », langage primitif et véridique, qui sera si cher aux dramaturges sensibles du XVIII ${ }^{\mathrm{e}}$ siècle, soucieux de restaurer à l'elocutio dramatique davantage de naturel. Le rapport au discours permet, une fois de plus, d'opposer héros sensibles et cœurs froids, dans la mesure où les paroles de Plautie traduisent la vérité et la plénitude du sentiment, alors que celles d'Appius ne sont que des formules creuses. À travers ce face à face se fait jour l'idée que la virtuosité rhétorique est suspecte et que, fruit d'une élaboration dénaturante ${ }^{34}$, elle révèle bien souvent l'insensibilité du locuteur.

La confrontation d'Appius et de Plautie permet de faire tomber les masques et révèle le véritable visage du tyran en même temps que la cause des malheurs des héros. Appius se trouve disqualifié, non seulement en tant qu'individu mais surtout en tant que représentant d'un pouvoir illégitime et d'une pratique politique corrompue. Dès lors, tout l'effort des héros consistera à remédier à cette anomalie éthique (la souffrance d'un innocent) et politique (la perversion d'un pouvoir mis au service du vice) ${ }^{35}$. Les larmes, signes jusqu'alors de l'impuissance de Plautie, vont se retourner en outil de conquête du pouvoir et assurer le rétablissement d'un ordre légitime, en même temps que la victoire des valeurs sensibles.

$\mathrm{Au}$ contraire du tyran, les cœurs vertueux se montrent en effet accessibles à la pitié et la compassion les porte naturellement à soulager la douleur d'autrui. L'empathie a donc pour corollaire immédiat l'action. C'est le

\footnotetext{
33 Campistron, acte III, scène 2, p. 49.

34 Voir sur ce point les théories diderotiennes et les reproches adressés à Corneille par Voltaire qui, dans ses Commentaires, juge que ses héros parlent en rhéteurs quand ils devraient paraitre passionnés. Le langage pathétique, chez les dramaturges sensibles du XVIII ${ }^{e}$ siècle doit nécessairement, pour être reçu comme authentique, porter la marque de l'émotion et du naturel le plus sincère.

35 Au-delà de l'aberration politique que représente l'attitude d'Appius, c'est tout un modèle anthropologique qui se voit mis en cause par Plautie qui, dans sa douleur et sa fureur révolutionnaire, incrimine jusqu'aux dieux, coupables selon elle de cautionner l'injustice faite à sa fille. Elle raconte ainsi avec amertume, à la scène 1 de l'acte III (p. 46) : «Et quand pour adoucir un sort si rigoureux/ Pleine de désespoir je cours, je vole au temple,/ Hélas! Par un destin qui n'eut jamais d'exemple/ Cet asile sacré contre tous nos malheurs/ Qui toujours des humains soulage les douleurs,/ La présence des dieux irrite ma disgrâce/ Puisque mes tristes yeux y remarquent la place/ Où ces dieux ont permis que des monstres cruels/ M'aient ravi ma fille au pied de leurs autels ». Cette mise en cause des dieux sonne comme un appel à une humanisation des figures religieuses, qui se verra accomplie dans le théâtre des Philosophes.
} 
cas pour Icile ${ }^{36}$. Mais c'est surtout chez Plautie que cette transformation de la souffrance passive en activité protectrice est remarquable. Campistron assigne en effet à ce personnage une importance inédite ${ }^{37}$. Plus qu'Icile dont les velléités de soulèvement populaire se voient entravées par son emprisonnement, plus que Virginius que Campistron maintient hors de la scène et de Rome jusqu'au dernier moment, c'est Plautie qui, transformant sa douleur en fureur, va inciter le peuple à prendre le parti de Virginie et à se révolter contre Appius. Loin de se résigner ou de compter sur une intervention providentielle, elle entreprend d'agir par elle-même pour sauver sa fille, déclarant dès la scène 4 de l'acte I :

Enfin, pour te sauver, il suffira de moi ;

Que ne pourrai-je point en agissant pour toi ?

Nous attendons beaucoup du secours de leurs armes:

Mais n'espère pas moins de celui de mes larmes ;

De cet affreux palais, j'ouv rirai les chemins

Je servirai de chef aux premiers des Romains

Et mes brûlants soupirs verseront dans leur âme

Cette bouillante ardeur qui m'anime et m'enflamme. 38

L'assimilation des larmes aux armes et la mise sur un pied d'égalité de la force militaire incarnée par Icile et Virginius avec la puissance persuasive du spectacle de la douleur, en même temps qu'elles posent l'existence d'une force des faibles, suggèrent la possibilité d'une action collective et donc d'une pratique politique fondée non sur les lois de l'univers épique mais sur les principes éthiques de la sensibilité. Campistron postule que la compassion et l'amour de la vertu unissent tout aussi efficacement et plus naturellement les hommes que les lois de l'honneur ou des codes de la politique romaine, tout en apportant la garantie d'une plus grande justice. Avec Plautie, c'est donc l'avènement d'un modèle politique gouverné par la sensibilité et le mode de sociabilité harmonieuse qu'elle induit qui se produit au théâtre et vient régénérer le genre de la tragédie romaine. Campistron fait dire à son héroïne, dans une réplique révélatrice de sa propre activité esthétique :

Oui, je veux que mes cris

Réveillent la vertu des Romains assoupis.

Je veux leur inspirer les transports de mon âme.

Sans doute, ils rougiront en voyant une femme

Moins timide cent fois et plus romaine qu'eux

\footnotetext{
36 qui, à la scène 2 de l'acte II, dit à Virginie : « Madame, par pitié, cachez-moi vos douleurs./ C'est trop de mes ennuis et de votre tristesse./ [...] Je perdrai vos tyrans et quel que soit leur rang/ Ces pleurs que vous versez leur coûteront du sang » (p. 35).

${ }^{37}$ Voir ce qu'en dit Lancaster, dans $A$ History of French dramatic literature in the seventeenth-century. Part IV : the period of Racine (1673-1700). 1940, The Johns Hopkins Press / Les Belles Lettres, t. I, p. 246.

${ }^{38}$ Campistron, acte I, scène 4, p. 23.
} 
Tâcher de ranimer cet esprit généreux

Qu'a versé dans leur sein le sang de leurs ancêtres

Sans cesse révolté contre d'injustes maîtres. ${ }^{39}$

Cette pratique du pouvoir a quelque chose de révolutionnaire dans la mesure où elle réunit ceux qui sont traditionnellement exclus de la chose publique (femmes, simples citoyens membres de la foule généralement silencieuse et passive) en une collectivité soudée par les effets de l'humanité sensible. Le prosélytisme de Plautie fonctionne sur le mode de l'identification et de l'implication empathique. Si, au début de la pièce, le peuple se contentait, de compatir de loin " à la juste douleur/ D'un amant éperdu, d'une mère en fureur ${ }^{40} "$, tout l'effort de Plautie va désormais consister à fédérer les femmes de la cité en les impliquant activement dans les malheurs qui la touchent. Elle expose sa stratégie à Virginie, à la scène 7 de l'acte III :
Je vais par un récit des maux que je prévois
Faire trembler les cœurs des mères comme moi.
Je vais les alarmer pour toute leur famille
Par l'exemple inoui des malheurs de ma fille.
Je vais tout animer contre Appius, enfin,
Je cours périr moi-même ou changer ton destin. ${ }^{41}$

Le destin singulier de sa fille devient un enjeu collectif et le trouble à l'ordre domestique se transforme en affaire d'état ${ }^{42}$. Si elles ne parviennent pas à fléchir un tyran, les larmes d'une mère s'avèrent en revanche fort capables de lui opposer un contre-pouvoir et de soulever une armée de femmes susceptible de le faire vaciller. Campistron affirme en effet l'efficacité des larmes et nous montre, à la scène 7 de l'acte IV, Claudius s'inquiétant des réactions suscitées par les larmes de Plautie et mettant en garde Appius :

Craignez sa fatale douleur.

On la voit en tous lieux de Romaines suivie À tous nos citoyens demander Virginie.

Ces femmes, à l'envi, par de tristes accords Expriment leurs regrets en des termes si forts Qu'il semble que chacune ayant perdu sa fille Déplore les malheurs de sa propre famille.

Les unes par des pleurs exhalent leur courroux, D'autres pour animer le peuple contre vous Poussent jusques au ciel mille cris pitoyables.

\footnotetext{
${ }^{39}$ Campistron, acte III, scène 6, p. 62.

40 Campistron, acte I, scène 1, p. 7 .

${ }^{41}$ Campistron, acte III, scène 7, p. 65.

${ }^{42}$ C'est ce qu'explique Plautie à Virginie, à la scène 7 de l'acte III (p. 65) : «Tous les Romains, ta cause est la cause commune./ Il s'agit de leur sort comme de ta fortune./ Le perfide Appius a commencé par nous/ Mais demain sur quelque autre il portera ses coups./ Si tous nos citoyens armés pour ta défense/ N’assurent leur repos en vengeant notre offense ».
} 
Plusieurs pour éviter des disgrâces semblables

Embrassent leurs enfants et courent les cacher

Craignant que de leurs bras on les vienne arracher.

Enfin, à les sauver leur amitié s'empresse

Et la peur de les perdre augmente leur tendresse. ${ }^{43}$

L'originalité de Campistron est ici évidente : loin de former, comme chez TiteLive $^{44}$, un chœur voué à déplorer le sort de Virginie et de se réduire à un tableau pathétique, les romaines gagnées à la cause de Plautie par la seule contagion des larmes ${ }^{45}$ se constituent en véritable armée. Ce pouvoir inédit étonne Plautie elle-même à qui Pison, rapportant les agissements d'Appius, apprend :

Mais d'un péril trop grand il s'est vu menacé

Vos pleurs étaient plus forts que les armes d'Icile.

Déjà de toutes parts on voyait dans la ville

Les femmes à l'envi sur vos pas s'assembler

Plautie réplique, interloquée par la puissance des effets de la sympathie :

Quoi! Nos clameurs l'ont pu faire trembler!

Il craint notre douleur dont les plus fortes armes

N’ont été que des vœux, des soupirs et des larmes.

Mais voilà le destin des tyrans tels que lui,

Ils traînent avec eux un éternel ennui. ${ }^{46}$

43 Campistron, acte IV, scène 7 , p. 83.

${ }^{44}$ Les femmes sont en effet présentes dans le récit livien, mais demeurent spectatrices des efforts de Virginius et d'Appius pour sauver Virginie. Si compassion et pathétique se voient déjà attachés à la foule, celle-ci se montre en revanche relativement passive. Tite-Live raconte : «Lucius Verginius arriva en tenue de deuil avec sa fille, vêtue de haillons; quelques femmes les accompagnaient, ainsi qu'un nombre impressionnant de défenseurs. [...] Les simples pleurs des femmes qui les avaient accompagnés étaient plus émouvants que tous les discours» (p. 335). Les pleurs des femmes ponctuent le calvaire de l'hérö̈ne. Ainsi : «Marcus Claudius alla chercher la jeune fille au milieu des femmes qui l'entouraient, quand les femmes l'accueillirent par un concert de lamentations et de gémissements (p. 336) ». Le dénouement est explicite sur la charge pathétique de ce chœur féminin : «Icilius et Numitor enlevèrent le corps inanimé pour le montrer au peuple. Ils pleuraient sur le crime d'Appius, sur la beauté de la jeune fille qui avait causé son malheur, sur le funeste devoir d'un père. Les femmes [...] ajoutaient encore d'autres regrets que la douleur arrache aux femmes en de telles circonstances : le chagrin des femmes, d'autant plus violent qu'il est moins contrôlé, est particulièrement pathétique » (p. 338).

45 Fulvie dit en effet, à la scène 1 de l'acte III (p. 45) : «Madame, où courrez-vous ? Vous verrai-je toujours/ D'une douleur mortelle entretenir le cours?/ Sourde à tous nos conseils, désespérée, errante,/ Loin d'adoucir vos maux, chaque instant les augmente,/ Un chagrin dévorant précipite vos pas.../ [...] Aux discours des romains, touchés de vos malheurs/ Vous avez seulement répondu par des pleurs ».

46 Campistron, acte V, scène 1, p. 86. 
Au pouvoir illégitime du tyran, la sensibilité oppose un modèle positif qui réconcilie politique et humanité. La révolte des femmes assure, en proclamant l'efficacité des larmes, la promotion d'une vision du monde optimiste fondée sur les valeurs de la sensibilité.

La fable est, à cet égard symptomatique d'une évolution profonde de la tragédie qui se détourne des modèles esthétiques autant qu'anthropologiques incarnés par les dramaturgies de Corneille et Racine pour chercher une voie nouvelle, sans doute inspirée par l'opéra, afin d'adapter le genre aux attentes d'un public désormais féru d'un pathétique à visage humain. En ce sens, la tentation est grande de lire dans la place accordée aux larmes dans Virginie un propos métathéâtral implicite proposant un modèle de réception inédit, qui ne saurait se réduire à la conception traditionnelle de la catharsis mais situe la question de l'efficacité du théâtre du côté de l'action et de la contagion, aussi bien morale que politique. Par bien des aspects, Campistron annonce ainsi le théâtre sérieux du dix-huitième siècle. 
